\title{
Globular Clusters in Early-Type Galaxies with GMOS
}

Terry Bridges, Mike Beasley, Favio Faifer, Duncan Forbes, Juan Forte, Karl Gebhardt, Dave Hanes, Ray Sharples, Steve Zepf

AAO, Swinburne, UNLP/CONICET, Swinburne, UNLP/CONICET, U. of Texas, Queen's U., Durham, Michigan State U.

\begin{abstract}
We present recent results from our long-term Gemini/GMOS study of globular clusters (GCs) in early-type galaxies. To date, we have obtained photometry and spectroscopy for GCs in NGCs 3379, 4649, 524, 7332, and IC 1459. We find a clear bimodality in the NGC $4649 \mathrm{GC}$ color distribution, with the fraction of blue/red clusters increasing with galacto-centric radius. We derive ages and metallicities for 22 GCs in NGC 3379 , finding that most of the clusters appear old (10-15 Gyr); however, there is a group of 4 metal-rich, younger clusters with ages of 2-6 Gyr. The NGC 3379 GC velocity dispersion decreases with radius, as does the inferred (local) mass-to-light ratio: there is no evidence for a dark matter halo in NGC 3379 based on our GC data.
\end{abstract}

\section{Introduction}

Globular clusters (GCs) are excellent probes of the dynamics, dark matter content, star-formation histories, and chemical enrichment of early-type galaxies. We have embarked upon a major programme using the Gemini Multi-Object Spectrograph (GMOS) on the 8m Gemini telescopes to obtain photometry and spectroscopy of GCs in 12 early-type galaxies, covering a range of galaxy type, luminosity, and environment. In this paper we present some of the first results from this programme.

\section{Data}

We have been using GMOS on Gemini North (since 2002A) and South (since 2003B) to obtain spectroscopy for 30-100 GCs in each of NGCs 3379, 4649, 524, 7332, and IC 1459. GMOS pre-imaging in the g', r', and i' filters for 2-3 fields per galaxy (400-800 sec in each filter) is used to select GC candidates for followup spectroscopy and for photometric analysis of the GC systems. The GMOS images have been reduced using IRAF/DAOPHOT, with photometric calibration from HST photometry kindly supplied by Soeren Larsen. Final GC candidate lists are determined after rejection of resolved objects, and objects with colors outside the range of Galactic GCs. GMOS multi-slit spectroscopy has been obtained for one or more fields in each galaxy, with 25-50 slits per field/mask (depending on the richness of the GC system), and exposure times of $8-10$ hours per mask. Spectroscopic data reduction is done using the IRAF/GMOS package. 


\section{Globular Cluster Photometry in NGC 4649}

NGC 4649 is a luminous $\left(\mathrm{M}_{V}=-22.4\right)$ Virgo elliptical. We present preliminary photometric results for $\sim 2000$ unresolved objects with i' $<25$ and $0.5<\left(\right.$ g' $\left.^{\prime}-\mathrm{i}^{\prime}\right)$ $<1.5$ (see Forbes et al. (2004, MNRAS, 355, 608) for a fuller account, and Bridges et al. (2003, EGCS Conf. 6, 36) for NGC 4649 GC spectroscopy). The NGC 4649 GC colour distribution is clearly bimodal, with peaks at $\left(g^{\prime}-i^{\prime}\right)=\sim$ 0.9 and 1.15. The fraction of blue/red clusters increases with radius, as found in other early-type galaxies.

\section{Globular Cluster Spectroscopy in NGC 3379}

NGC 3379 is a less luminous elliptical in the nearby Leo group. We have spectra for 22 NGC 3379 GCs in a 10 hour GMOS exposure with $5 \AA$ resolution and coverage from $\sim 4000-7000 \AA$. Figure 1 shows the ages and metallicities of 18 GCs, based on the comparison of line-strengths with stellar population synthesis models. Most of the GCs are old, with ages $>10 \mathrm{Gyr}$, but there is a group of $~$ 4 metal-rich, younger GCs with ages of 2-6 Gyr. Figure 2 shows that both the $\mathrm{GC}$ velocity dispersion (top panel) and the local M/L (bottom panel) decrease outwards. A preliminary analysis (see Beasley et al. 2004) shows that there is no evidence for a dark-matter halo in NGC 3379, a very interesting result also seen in the PNe kinematics (Romanowsky et al. 2003, Science, 301, 1696).
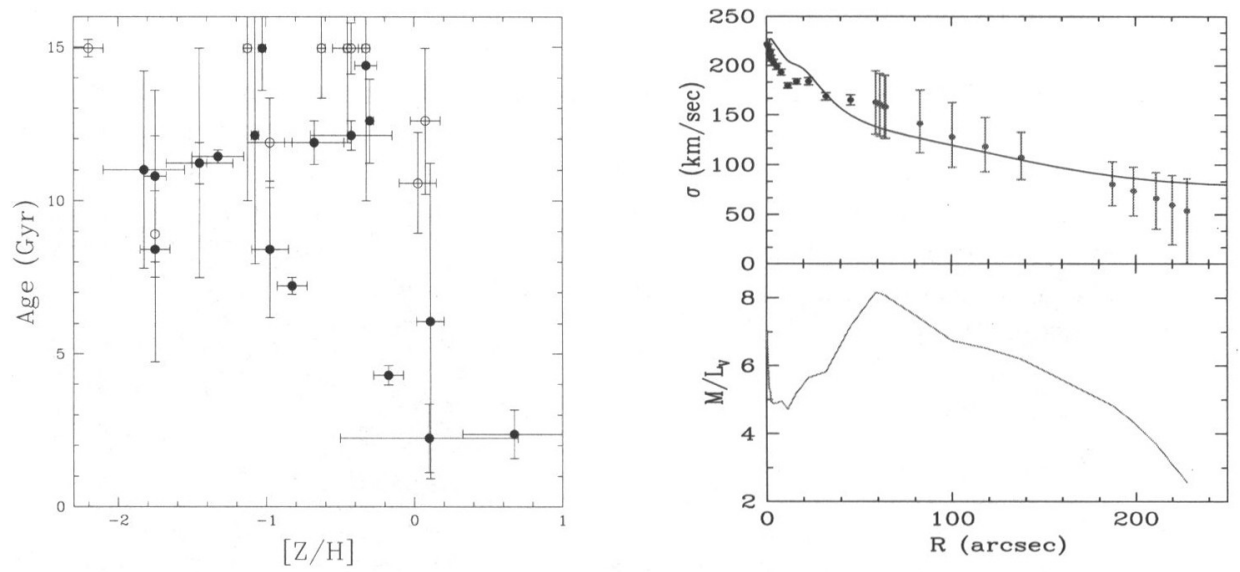

Figure 1 (left): Ages/metallicities for NGC 3379 GCs (filled circles) and MW GCs (open circles), from our GMOS spectra. Figure 2 (right): Top: velocity dispersion of NGC 3379 stars (small errors) and GCs (larger errors); the solid line is a constant M/L fit. Bottom: The local M/L for NGC 3379.

Acknowledgments. We are very grateful to Søren Larsen for all of his help with our photometric calibration, and to the Gemini staff, particularly Inger Jørgensen, for obtaining such nice data for us. 